\title{
Treatise on analytic optimal spacecraft guidance and control
}

\author{
Timothy Sands $1, *$
}

1 Sibley School of Mechanical and Aerospace Engineering, Cornell University, Ithaca USA; tas297@cornell.edu

\begin{abstract}
Autonomous navigation of spacecraft necessitates innovative technologies, methods, and algorithms, particularly when orbiting in proximity of other space objects. Optimization methods are useful for autonomous spacecraft navigation, guidance, and control, but their performance is hampered by noisy multi-sensor technologies and poorly modeled system equations, and real-time on-board utilization is generally computationally burdensome. Some proposed methods use noisy sensor data to learn the optimal guidance and control solutions real-time (online), where non-iterative instantiations are preferred to reduce computational burdens. This study aims to highlight efficacy and limitations of several common methods for optimizing guidance and control while proposing a few more, where all methods are applied to the full, nonlinear, coupled equations of motion including cross-products of motion from the transport theorem. Five disparate types of optimum guidance and control algorithms are presented and compared to a classical benchmark. Comparative analysis is based on tracking errors (both states and rates), fuel usage, and computational burden. Real-time optimalization with singular switching plus nonlinear transport theorem decoupling proves superior by matching open-loop solutions to the constrained optimization problem (in terms of state and rate errors and fuel usage), while robustness is validated in the utilization of mixed, noisy state and rate sensors and uniformly varying mass and mass moments of inertia. State tracking errors are reduced one-hundred ten percent. Rate tracking errors are reduced one-hundred thirteen percent. Control utilization (e.g., fuel) is reduced eighty four percent, while computational burden in reduced ten percent simultaneously.
\end{abstract}

Keywords: autonomy; optimal; spacecraft; navigation guidance; attitude control; inertial sensors; star trackers; linear quadratic regulator; time-optimal control; optimal open loop; proportional plus derivative control; real-time optimal control; switched real-time optimal control

\section{Introduction}

Considering lack of coverage and Earth-to-space communication delays, autonomous guidance and control necessitates real-time on-board computation with demanding accuracy and robustness requirements, despite potentially coarsely known spacecraft characteristics, varying environmental conditions and mission-related constraints.

Many solutions have been developed [3] including optimal analytic methods for simple cases while optimal methods guiding and controlling realistic nonlinear space systems ubiquitously require computational solutions. This manuscript proposes new techniques for utilizing optimization techniques applied to the full, nonlinear, coupled equations of spacecraft motion, and the techniques are analytic as opposed to numeric. Rao proposed numerical trajectory optimization applied to orbital transfer problems [4] but also produced a survey of numerical methods for optimal control [5]. Numerical methods are very quicky resorted to as researchers grapple with six nonlinear, coupled equations of mechanical motion (both translation and rotation). A generalized treatment method (again numerical) as optimization problems was proposed in [6] for such orbital transfer problems, spacecraft rendezvous and docking [7-9], and planetary entry and hypersonic space planes [10-14]. Arguably, following the publication of [15], numerical optimization in general form realized the current dominance of numerical methods, e.g. 
$[16,17]$ for real-time (numerical) trajectory optimization, $[18,19]$ for aero-assisted optimal tracking guidance.

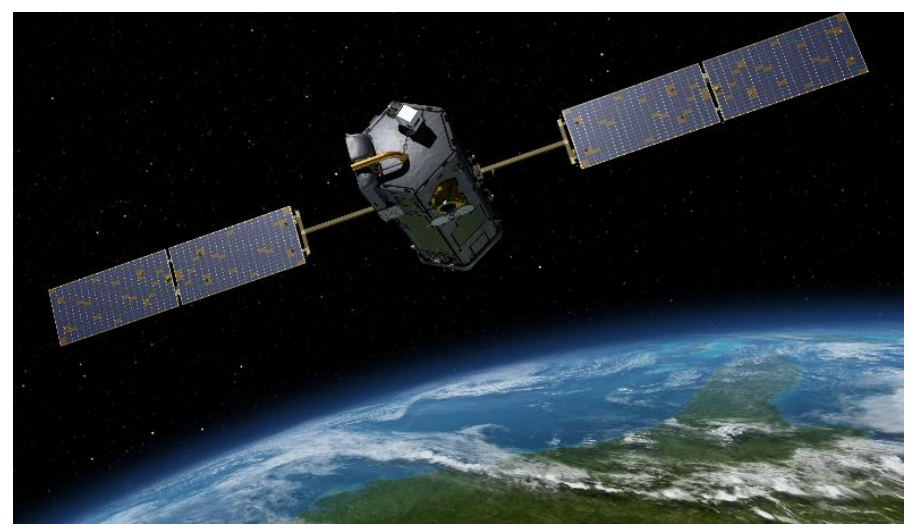

Figure 1. The Applied Remote Sensing Training Program (ARSET) for measuring and monitoring changes in vegetation using solar-induced fluorescence and LiDAR. [1] Image used consistent with NASA policy, "NASA content (images, videos, audio, etc) are generally not copyrighted and may be used for educational or informational purposes without needing explicit permissions." [2].

Lacking ubiquitous analytic methods to treat the nonlinear, coupled systems of equations, linearization followed by least squares optimization leads to so-called Ricatti equations [20] to produce optimal control gains [21] with a presumed error feedback [22] in both continuous and discrete form [23]. Optimization is sometimes sought after first implementing adaptive [24] methods to use feedback achieving predictability [25]. Championed by Lorenz, physics-based methods [26] were proposed to instantiate "selfsensing machines methods, where the sensing functions are fully integrated on a drive to detect key operating characteristics including rotor position, torque, speed, temperature and motor/load diagnostics.". [27] The physics-based methods codified optimal feedforward forms which were later augmented with optimal feedback [28] instantiating the relatively new method referred to as deterministic artificial intelligence (D.A.I.). D.A.I. necessitates analytic forms of desired state trajectories [29] for the feedforward control and state observers [30] for the feedback control. In 2021, utilization of Pontryagin's approach $[31,32]$ to impose necessary conditions of optimality as a first step led to boundary-value problems that produce optimal controls, but also optimal trajectories [33] as alternatives to the sinusoidal trajectories recommended by Baker [29].

Thus, the reader may consider use of classical control methods (proportional plus velocity will be evaluated here) and seek to optimize control gains or use the Ricatti equation to seek linear-quadratic optimal classical control gains. Alternatively, timeoptimal control may be considered as a feedback form or control-minimizing control in an open-loop feedforward topology. Still further, real-time optimal controls could be derived that utilize feedback of state and rate in a matrix-inverse to enforce optimality in closed-loop. Several different options for matrix inversion are available generating subsets of the broader category of real-time optimal control. A key limitation of all the methods described so far is the inability to deal with nonlinear, coupled equations generated by the transport theorem in both translational and rotational mechanics.

Next-generation methods are required that achieve mathematically optimal results yet retain the simplicity of analytics solutions obfuscating numerical methods providing further advancements in autonomous navigation. The current movement towards utilization of very small space vehicles is accompanied by very limited computational resources, while maintaining autonomy, robustness, and accuracy. Newly proposed methods and algorithms for autonomous navigation must be presented in direct, critical comparison to the recent research trends of both academia and industry, presuming utilization of noisy sensors, e.g., star trackers, rate gyroscopes, inertial measurement units, and global navigation systems, amongst other sensors in multi-sensor-based architec- 
tures for spacecraft navigation. Intelligence methods permitting spacecraft to learn realtime optimal solutions (analytically) are preferred.

Proposed novelties:

1. Brief methodological recitation of five disparate incarnations of optimal control and their direct comparison to classical feedback control as a benchmark (the $\mathrm{P}+\mathrm{V}$ proportional plus velocity controller): 1) control-minimizing open-loop optimal, 2) linear-quadratic optimal regulator, 3) time-optimal, 4) real-time optimal, and 5) realtime optimal with singular switching.

2. Direct comparison of the efficacy of each of the six methods listed in item \#1 controlling double-integrator plants, where comparison is made using state accuracy, rate accuracy, control (e.g., fuel) usage, and computational run-time (as a manifestation of computational burden);

3. Direct comparison of the efficacy of each of the six methods listed in item \#1 controlling double-integrator plants including transport theorem cross-products of motion induced by measurement in rotating reference frames, where comparison is made using state accuracy, rate accuracy, control (e.g., fuel) usage, and computational run-time (as a manifestation of computational burden). In item \#3, the linear control designed are used on the nonlinear plants to evaluate the error resulting from using linear control designs in the real-world on nonlinear systems.;

4. Direct comparison of the efficacy of each of the six methods listed in item \#1 controlling double-integrator plants including transport theorem cross-products of motion induced by measurement in rotating reference frames, where comparison is made using state accuracy, rate accuracy, control (e.g., fuel) usage, and computational run-time (as a manifestation of computational burden). Unlike item \#3 above, nonlinear decoupling control stemming from the solution to the minimum-control optimization problem is introduced to each control methodology by utilizing the optimal rate trajectories that result from the original open-loop optimization problem that minimizes control effort.

5. Items \#3 and \#4 are both repeated to evaluate the deleterious effects on each method of noisy sensors and random uniformly varying spacecraft mass and mass moments of inertia.

Section 2 includes very brief derivations of each respective approach as briefly as practicable, while section 3 provides the results of implementing each disparate methodology.

\section{Materials and Methods}

Motion (both translational and rotational) is governed by so-called doubleintegrator dynamics where the integral of applied forces vector (inversely scaled by the mass or mass moments respectively) is the velocity vector and the integral of the velocity vector (translational or rotational) is the displacement vector. Each vector is relative to an inertial (non-rotating) reference frame, while expression of the vectors in the coordinates of the basis vectors of rotating reference frames necessitates inclusion of the transport theorem which articulates the induced motion of the rotating reference frame in cross-products that make the results nonlinear and coupled. The three degrees of rotational motion are coupled to each other by the transport theorem, and the three degrees of translational motion are coupled to each other as well. Furthermore, the three degrees of translation are coupled nonlinearly to the three degrees of rotation, particularly through the angular velocity vector. Especially since this nonlinear coupling is a foremost challenge that is often deemed insurmountable by analytic methods, the foremost subsections of this part of the manuscript begin so. The Materials and Methods section of the manuscript is described with sufficient details to allow readers to replicate and build on the published results. 
Table 1. Proximal variable definitions.

\begin{tabular}{|c|c|c|c|}
\hline Variable & Definition & Variabl & Definition \\
\hline$F$ & Externally applied forces & $T$ & Externally applied torques \\
\hline$m$ & Mass & $J$ & Mass moment of inertia \\
\hline$a=\ddot{x}$ & Translational acceleration & $\alpha=\ddot{\theta}$ & Rotational acceleration \\
\hline$\frac{d^{2} x}{d t^{2}}=\dot{v}$ & Translational acceleration & $\frac{d^{2} \theta}{d t^{2}}=\dot{\omega}$ & Rotational acceleration \\
\hline$r$ & Radius vector relative to rotating frame & $\omega=\dot{\theta}$ & Rotational velocity \\
\hline$v$ & Velocity vector relative to rotating frame & $\theta$ & Displacement angle \\
\hline
\end{tabular}

${ }^{1}$ Such tables are distributed throughout the manuscript to increase the ease of reading, while a combined, master table of definitions in included in the appendices.

\subsection{Double-integrator based plant equations}

Equation (1) illustrates the fundamental relationships of both translational and rotational motion may be expressed as so-called "double-integrators" meant to mean the twice integration of the applied force or torques produces the respective translational or rotational displacement.

$$
F=m a=m \ddot{x}=m \frac{d^{2} x}{d t^{2}} \leftrightarrow T=J \alpha=J \dot{\omega}=J \ddot{\theta}=J \frac{d^{2} \theta}{d t^{2}}
$$

Equation (1) comprises two sets of three equations each for translation and rotation combining for six equations of mechanical motion. For simplicity of expression, states referred to generically as $x$ can represent rotations $(\theta)$ with regards to the basic, shared motion described by the double-integrator. Transport theorem described next will generate differences in the governing equations for translation and rotation.

\subsection{Transport theorem cross-product coupled motion expressed in rotating reference frames}

Attach three mutually perpendicular unit vectors to each frame: non-rotating inertial frame and body-fixed frame. The meaning of differentiation of vectors when specification is made of differentiation with respect to a specific frame. Both rotational and translational motion relative to the non-rotating reference frame may be represented by double integrators in accordance with equation (2).

$$
\left.m a\right|_{\text {relative }}=\left.m \ddot{x}\right|_{\text {relative }}=\left.m \frac{d^{2} x}{d t^{2}}\right|_{\text {relative }}
$$

Theorem 1. Transport Theorem. The derivative of any vector expressed in the coordinates of a rotating reference frame equals the sum of the derivative relative to a non-rotating reference frame plus the cross-product of the angular velocity and the vector.

Proof of Theorem 1. The proof of this well-known theorem is provided by citation [34]. The tedious process may be summarized: 1 ) express the position vector with respect to the non-rotating inertial reference frame; 2 ) differentiate to find the expression for velocity remembering to differentiate both the component measurements and the unit vectors; 3) simplify and substitute the defined unit vectors, define the angular velocity in the direction perpendicular to the two-dimensional space of rotation; 4) substitute the newly defined angular velocity to arrive at the Transport Theorem as expressed in equation (3). $\square$

$$
J \frac{d \omega}{d t}=\left.J \frac{d \omega}{d t}\right|_{\text {relative }}+\omega \times J \omega
$$




\subsubsection{Euler's moment equations of rotation expressed in a rotating reference frame}

Externally applied torques, $T$ change angular momentum $J \frac{d \omega}{d t}$ permitting the substitution into equation (3) resulting in Euler's moment equation in equation (4).

$$
T=J \dot{\omega}+\omega \times J \omega
$$

Notice the dominant double-integrator dynamics are embodied in $J \dot{\omega}$ in equation (4) while the additional accelerations due to the transport theorem are embodied in the coupling cross-product term $\omega \times J \omega$. Control designs based on the double integrator dynamics alone are hypothesized to have less efficacy than proposed techniques that utilize optimization and the transport theorem terms.

\subsubsection{Newton's equations of translation expressed in a rotating reference frame}

Performing similar expression of translational motion in non-rotating inertial frames as just performed in section 2.2.1 for rotational motion leads to equation (5) for Newton's equations of translational motion.

$$
F=\underbrace{m a}_{\text {Relative }}+\underbrace{m \frac{d \omega}{d t} \times r}_{\text {Euler }}+\underbrace{2 m \omega \times v}_{\text {Coriolis }}+\underbrace{m \omega \times \omega \times r}_{\text {Centrifigual }}
$$

Notice the dominant double-integrator dynamics relative to the rotating reference frame in equation (5) are embodied in $m a$ while the additional accelerations due to the transport theorem are embodied in the coupling cross-product terms: Euler $\left(m \frac{d \omega}{d t} \times r\right)$, Coriolis $(2 m \omega \times v)$, and centrifugal $(m \omega \times \omega \times r)$. Control designs based on the double integrator dynamics alone are hypothesized to have less efficacy than proposed techniques that utilize optimization and the transport theorem terms.

Neglecting the cross-products of acceleration resulting from the Transport Theorem reduces both equations (4) and (5) to the double integrators of equations (1) and (2). The goal of this research is to develop controls (for applied forces $F$ and applied torques $T$ ) that account for the nonlinear, coupling cross-products produced by application of the Transport Theorem.

\subsection{Classical position plus velocity $(P+V)$ feedback control}

Proportional plus velocity control [1] utilizes proportional control by forming a state error scaled by a proportional gain adding a negative gained value of velocity (translational or rotational) as elaborated in equation (7). The velocity channel is not a differentiated version of the position or angle channel, as is the case with classical cascaded control topologies of PD, PI, PID types (proportional plus derivative, proportional plus integral, and proportional plus integral plus derivative respectively).

Table 2. Proximal variable definitions.

\begin{tabular}{cccc}
\hline Variable & Definition & Variable & Definition \\
\hline$x_{d}$ & Desired state trajectory & $\xi$ & Critical damping ratio \\
$K_{P}$ & Proportional gain & $\omega_{n}$ & Natural frequency \\
$K_{v}$ & Velocity gain & $t_{s}$ & Settling time \\
\hline
\end{tabular}

${ }^{1}$ Such tables are distributed throughout the manuscript to increase the ease of reading, while a combined, master table of definitions in included in the appendices.

$$
\begin{gathered}
x(s) \\
\frac{x\left(\ddot{x}+K_{V} \dot{x}+K_{P} x=K_{P} x_{d} \leftrightarrow u=K_{p}\left(x_{d}-x\right)-K_{v} \dot{x}\right.}{x_{d}(s)}=\frac{K_{p}}{J s^{2}+K_{v} s+K_{p}} \rightarrow \text { C.E. }: s^{2}+K_{v} s+\left.K_{p}\right|_{I=1}=s^{2}+2 \xi \omega_{n} s+\omega_{n}^{2}
\end{gathered}
$$

Gains were tuned for performance specification by equating the ubiquitous closedloop system equation (5) to the performance specified. The desired rise time established 
the system natural frequency per $t_{r}=\frac{1.8}{\omega_{n}}$ where $\omega_{n} \approx \omega_{b}$ is the desired control bandwidth, therefore $\omega_{n}=\frac{1.8}{t_{r}} \rightarrow K_{p}=\omega_{n}{ }^{2}$. Settling time: oscillation stabilize within 2-5\% percent of steady state $t_{s}=\frac{4.6}{\xi \omega_{n}} \rightarrow \xi=\frac{4.6}{t_{s} \omega_{n}} \rightarrow K_{v}=2 \xi \omega_{n}$.

Elimination of differentiation in the derivative channel often bestows relative advantage tracking desired velocity trajectories. Another approach is the optimize gain selection, and this alternative approach is called the linear quadratic regulator.

\subsection{Linear-quadratic optimal regulator $(L Q R)$ of proportional derivative (PD) type [35]}

Equations (3) and (4) representing the full, nonlinear, coupled equations of motion in six degrees may be linearized and be expressed in the form displayed in equation (6). This linearization is the basis for the word "linear" in the LQR title. The word "quadratic" $^{\prime \prime}$ refers to selecting gains $K$ that minimize a quadratic cost function displayed in equation (7). The LQR solution [20,21] only bestows optimal solutions for control gains of the form equation (8) that minimize the quadratic cost simultaneously satisfying the (linearized) dynamic constraints displayed in equation (5).

$$
\begin{gathered}
\dot{x}=A x+B u \\
J=\int_{0}^{\infty}\left(x^{T} Q x+u^{T} R u\right) d t \\
u=-K x
\end{gathered}
$$

The control designer may select the state weighting matrix $Q$ and the control weighting matrix $R$ to respectively penalize the state errors and the control effort. In section 3, equally weighted identity matrices were chosen for both $Q$ and $R$. This choice facilitates a multi-faceted comparison in section 3 that does not solely focus on tracking errors or costs. The gains $K$ are found using equation (9) where the matrix $P$ is first found by solving the algebraic relation in equation (10) often referred to a Riccati equation which is most often solved iteratively by a computer (e.g., the MATLAB ${ }^{\circledR} /$ SIMULINK $^{\circledR}$ lqr command).

$$
\begin{gathered}
K=R^{-1}\left(B^{T} P\right) \\
A^{T} P+P A-P B R^{-1} B^{T} P+Q=0
\end{gathered}
$$

Table 3. Proximal variable definitions.

\begin{tabular}{cccc}
\hline Variable & Definition & Variable & Definition \\
\hline$A$ & State transition matrix & $J$ & Cost function \\
$B$ & Control matrix & $t_{f}$ & Final time \\
$K$ & Gain matrix & $\infty$ & Infinity \\
$Q$ & State weighting matrix & $s g n$ & Signum function \\
$R$ & Control weighting matrix & $p(t)$ & Parameters (co-states) \\
$P$ & Covariance matrix & $b_{i}$ & Control coefficients \\
\hline
\end{tabular}




\subsection{Time-optimal control [35]}

Minimizing a non-quadratic cost function comprised of only the final time (as displayed in equation (11)) constrained with the linearized dynamics of equation (6) with parameters $p(t)$ leads to time-optimal control [23-32].

$$
\begin{gathered}
J=\int_{0}^{\infty} t_{f} d t \\
u=\operatorname{sgn}\left(\left\langle p(t), b_{i}\right\rangle\right)=\left\{\begin{array}{cc}
1 & \text { if }\left\langle p(t), b_{i}\right\rangle>0 \\
-1 & \text { if }\left\langle p(t), b_{i}\right\rangle<0 \\
0 & \text { if }\left\langle p(t), b_{i}\right\rangle=0
\end{array}\right.
\end{gathered}
$$

Simulation subsystems depicted in the appendix execute a bang-bang control where maximal application of control is normalized to unity such that desired unity state and unity time is achieved to aid comparison to the other optimization approaches.

\subsection{Open loop minimum-control optimization $[15,31]$}

Minimizing only the control effort alone (not the state errors) [25] in accordance with equation (13) constrained by the double-integrator dynamics of equation (2) for specified initial and final conditions permits solution of a two-point boundary value problem producing optimal control, acceleration, rate, and state profiles displayed in equation (14) respectively. Normalization for unity masses or mass moments is included, thus control and acceleration equations are identical, where non-normalized control may be expressed by scaling the control equation by the masses or mass moments respectively.

By specifying quiescent initial conditions and using variable scaling and balancing to normalize the final position coordinate to unity, the constants in equation (14) may be solved resulting in equation (15), where $a=-12, b=6$, and $c=d=0$. Notice states are not penalized in the cost function, instead only solution forms that satisfy the boundary values are produced by the two-point boundary value problem from the initial point $(x(0), v(0))=(0,0)$ to the final point $(x(1), v(1))=(1,0)$, thus there is no need to solve an algebraic Riccati equation to produce the optimal control, where an additional benefit of this optimization approach includes the production of optimal state trajectories that will prove useful to decouple the nonlinear coupling effects of the transport theorem described in section 2.2. Scaling and balancing must be performed to normalize the initial and final conditions to zero and unity, and the operations are explained in section 2.11.

$$
\begin{gathered}
J=\frac{1}{2} \int_{0}^{\infty}\left(u^{T} u\right) d t \\
u=a t+b, \dot{v}^{*}=a t+b, v^{*}=\frac{1}{2} a t^{2}+b t+c, x^{*}=\frac{1}{6} a t^{3}+\frac{1}{2} b t^{2}+c t+d \\
u=-12 t+6, \dot{v}^{*}=-12 t+6, v^{*}=-6 t^{2}+6 t, x^{*}=-2 t^{3}+3 t^{2}
\end{gathered}
$$

Table 4. Proximal variable definitions.

\begin{tabular}{cccc}
\hline Variable & Definition & Variable & Definition \\
\hline$J$ & Cost function & $u^{*}$ & Optimal control \\
$d t$ & Differential time & $\dot{v}^{*}$ & Optimal (angular) acceleration \\
$T$ & Time & $v^{*}$ & Optimal (angular) velocity \\
$U$ & Control & $x^{*}$ & Optimal (angular) position \\
$x(t)$ & Current position & $a, b, c, d$ & Integration constants \\
$v(t)$ & Current velocity & $\hat{a}, \hat{b}, \hat{c}, \hat{d}$ & Integration constant estimates \\
\hline
\end{tabular}




\subsection{Real-time optimal control}

A corollary is to the open-loop minimum-control optimization in section 2.6 augments the approach with feedback while maintaining the remaining portions of the problem approach. The solution for the constants in between equations (14) and (15) may be accomplished real-time using feedback but asserting the current position and velocities (translational and rotational) are the initialization points of a new two-point boundary value problem. Equation 16 may be written in matrix-vector form as equation (17) permitting real-time solution for the integration constants in the vector by inverting the matrix and pre-multiplying both sides of the equation as depicted in (17). Notice the form of the control derived in equation (17) is the same as equation (14) in section 2.6, where the constants in the optimal solutions are solved real-time.

$$
\begin{aligned}
& v^{*}=\frac{1}{2} a t^{2}+b t+c, x^{*}=\frac{1}{6} a t^{3}+\frac{1}{2} b t^{2} \& v^{*}\left(t_{f}\right)=\frac{1}{2} a+b=0, x^{*}\left(t_{f}\right)=\frac{1}{6} a+\frac{1}{2} b=1 \\
& \underbrace{\left[\begin{array}{cccc}
\frac{t_{0}^{2}}{6} & \frac{t_{0}^{2}}{2} & t_{0} & 1 \\
\frac{t_{0}^{2}}{2} & t_{0} & 1 & 0 \\
\frac{1}{6} & \frac{1}{2} & 1 & 1 \\
\frac{1}{2} & 1 & 1 & 0
\end{array}\right]}_{[T]} \underbrace{\left\{\begin{array}{l}
a \\
b \\
c \\
d
\end{array}\right\}}_{\{p\}}=\{\underbrace{\left\{\begin{array}{c}
x_{0} \\
v_{0} \\
1 \\
0
\end{array}\right\}}_{\{b\}} \rightarrow\left\{\begin{array}{c}
\hat{a} \\
\hat{b} \\
\hat{c} \\
\hat{d}
\end{array}\right\}=\left[\begin{array}{cccc}
\frac{t_{0}^{2}}{6} & \frac{t_{0}^{2}}{2} & t_{0} & 1 \\
\frac{t_{0}^{2}}{2} & t_{0} & 1 & 0 \\
\frac{1}{6} & \frac{1}{2} & 1 & 1 \\
\frac{1}{2} & 1 & 1 & 0
\end{array}\right]^{-1}\left\{\begin{array}{c}
x(t) \\
v(t) \\
1 \\
0
\end{array}\right\} \text { and } u^{*} \equiv \hat{a} t+\hat{b},
\end{aligned}
$$

One key feature of the open loop solution method using a two-point boundaryvalue problem is enforcement of end conditions producing optimal trajectories for state $\left(x^{*}\right)$, rate $\left(v^{*}\right)$, acceleration $\left(\dot{v}^{*}\right)$, jerk $\left(\ddot{v}^{*}\right)$, etc. in addition to the formulation of an optimal control, $u^{*}$. These signals yield the opportunity to formulate decoupling control components to mitigate the transport theorem (to be illustrated in section 2.9).

\subsection{Real-time optimal control with singular switching}

Highlighting the matrix inverse in equation (17), the possibility of issues inverting a poorly conditioned or rank-deficient matrix may be addressed by monitoring matrix conditioning or determinant and switching away from the feedback solution when encountering rank-deficient instances in favor of the optimal solution in equation (15).

\subsubsection{Matrix inverse formulas}

Five disparate methods to invert the $[T]$ matrix were investigated as listed in equation (18). Matrix inversion methods already coded in MATLAB/SIMULINK: $[T]^{-1}$, $1 \backslash[T], \operatorname{inv}[T], \operatorname{pinv}[T]$, LU Inverse $[T]$. Each method has specific strengths and weaknesses expressed in state error, rate error, control effort (quadratric cost), and runtime as displayed in table 5 . In several instances, the simulation would fault as a result of encountering matrix singularity.

Table 5. Double-integrator plant ten-run mini-Monte Carlo analysis (faults occurred after first simulation run) executed in MATLAB $^{\circledR} /$ SIMULINK $^{\circledR}$ R2021b (9.11.0.1769968) whose machine precision eps $=2.2204 \times 10^{-16}$.

\begin{tabular}{ccccc}
\hline Method $^{1}$ & State Error & Rate Error & Cost & Runtime \\
\hline$[T]^{-1}$ & Fault & Fault & Fault & Fault \\
$1 \backslash[T]$ & 0.000052 & -0.0048598 & 4.0281 & 1.6221 \\
$\operatorname{inv}[T]$ & Fault & Fault & Fault & Fault \\
pinv[T] & 0.001169 & -0.003941 & 4.0281 & 1.5589 \\
LU Inverse[T] & Fault & Fault & Fault & Fault \\
\hline
\end{tabular}

${ }^{1}$ Real-time optimal control (with singular switching). 
Table 6. Proximal variable definitions.

\begin{tabular}{cccc}
\hline Variable & Definition & Variable & Definition \\
\hline$[T]^{-1}$ & MATLAB inverse & $m$ & Mass \\
$1 \backslash[T]$ & MATLAB inverse & $J$ & Mass moments \\
$i n v[T]$ & MATLAB inverse & $\omega$ & Angular velocity \\
$\operatorname{pinv}[T]$ & MATLAB Pseudo-inverse & $r$ & Position vector \\
$l u([T])$ & MATLAB LU-inverse & $v$ & Translational velocity \\
$u_{\text {transport }}$ & Feedback decoupler & $\omega^{*}$ & Optimal angular velocity \\
$u_{\text {transport }}^{*}$ & Feedforward (decoupler) & $\mathrm{r}^{*}$ & Optimal position vector \\
& & $\mathrm{v}^{*}$ & Optimal Translational velocity \\
$u$ & \multicolumn{2}{c}{ Control, the sum of equations (18) or (19) with (5), (8), (12), (14), or (15) } \\
\hline
\end{tabular}

\subsection{Nonlinear transport theorem decoupling (recall section 2.2)}

Section 2.2 describes nonlinear coupling effects of measuring motion in coordinates of rotating reference frames extracted for highlighting in equation (18) for translation and rotation respectively. These effects were neglected when optimizing the double integrator-based systems of equations or simplified by linearization in other instantiations. Taking advantage of the results in sections 2.6 and 2.7, nonlinear decoupling control components may be formulated using the optimal trajectories as displayed in equations (18-19), where each component (translation and rotation respectively) should be added to augment the control in equation (17).

$$
\begin{aligned}
& \left.\begin{array}{c}
\text { Rotation: } u_{\text {transport }}=\omega \times J \omega \\
\text { Translation: } u_{\text {transport }}=m \frac{d \omega}{d t} \times r+2 m \omega \times v+m \omega \times \omega \times r
\end{array}\right\} \text { Feedback } \\
& \left.\begin{array}{c}
\text { Rotation: } u_{\text {transport }}^{*}=\omega^{*} \times J \omega^{*} \\
\text { Translation: } u_{\text {transport }}^{*}=m \frac{d \omega^{*}}{d t} \times r^{*}+2 m \omega^{*} \times v^{*}+m \omega^{*} \times \omega^{*} \times r^{*}
\end{array}\right\} \text { Feedforward }
\end{aligned}
$$

\subsection{Noisy mixed sensors and parameter variations}

Especially with the inclusion of feedback, noise must be accounted for in the feedback signals (state and rate only here) in the form of random numbers with standard deviation of 0.01 ( $1 \%$ of the final state value when states are scaled and balanced to unity). In addition, mass and mass moments are assumed to be unknown or not precisely known, therefore mass and moments were allowed to vary randomly (uniformly) ten percent heavier and lighter. The resulting scatterplots will be presented in section 3 .

\subsection{Scaling and balancing}

Poorly conditioned problems are those requiring simultaneous mathematical operations on very large and small numbers. A common mitigation strategy is to scale and balance the variables transforming equations to nominally remain of the same order. Scaling problems by common, well-known values permits single developments to be broadly applied to a wide range of state spaces not initially intended. Normalizing time per equation (20) restricts simulation time to vary between zero and unity. Scaling mass and mass moments of inertia by their nominal values per equations (21) and (22) respectively keeps their values roughly on the order of unity. Generic displacements (translation or rotation) are normalized as well in accordance with equation (23) where $r$ indicates translational or rotational displacement.

$$
\begin{gathered}
t \equiv \frac{\bar{t}}{t_{f}} \\
m \equiv \frac{\bar{m}}{m_{\text {nominal }}}
\end{gathered}
$$




$$
\begin{gathered}
J \equiv \frac{\bar{J}}{J_{\text {nominal }}} \\
r \equiv \frac{\bar{r}}{r_{f}}
\end{gathered}
$$

\section{Results}

Following the just presented very brief introduction to each control technique in section 2, section 3 displays the results of individual simulations in addition to Monte Carlo investigation of ten-thousand simulations. Section 3.1 begins with commonly simplifying assumptions of control design using dominant, double-integrator dynamics with no transport theorem, where the control is applied to the same idealized system equations. The use of idealized results provides interesting measures of performance under ideal circumstances subject to mathematical optimization. Another interesting artifact is the immediately obvious differences in the responses to disparate control techniques.

Next, in section 3.2, the performance of controllers designed using simplified double-integrators were applied to more realistic plant equations with transport theorem. Then, in section 3.3 nonlinear control designs are introduced and comparisons are made applied to nonlinear plants including transport theorem. Lastly, in section 3.4 random uniformly varying inertia was studied with random noise added to sensor data for both state and rate, and the comparisons were repeated in ten-thousand simulations. These final simulations were all utilizing nonlinear control designs based on various optimization methods and the results were applied to nonlinear, coupled system equations including transport theorem, where controls were tailored specifically for the transport theorem in the recommended application of optimization (real-time optimal control with singular switching and transport theorem decouling). All simulations were executed in MATLAB $^{\circledast} /$ SIMULINK $^{\circledR}$ R2021b (9.11.0.1769968) whose machine precision eps $=2.2204 \times 10^{-16}$.

\subsection{Ideal, linear double integrator system equations}

Double integrator equations expressing relationships between displacement, displacement rate, and acceleration are canonical relationships used to describe movement of mass. The relationships are linear allowing easy control design using classical methods which predominantly use linear systems methods to design controllers for any equations (including linearizing any nonlinear equations). Each control design technique introduced in section 2 was sequentially used to control the linear, double integrator system equations, and the results are presented in figure 3 , where table 1 is embedded as a sub-figure containing quantitative results corresponding to the qualitative results presented in the multi-plots.

The baseline approach (classical proportional plus velocity, or $\mathrm{P}+\mathrm{V}$ ) tuned to performance specification exhibits better accuracy and lower costs than linear quadratic optimal regulators of the proportional, derivative (PD) type, but the $\mathrm{P}+\mathrm{V}$ controller has the highest computational burden as indicated by computational runtime. The embedded differentiation of the noisy feedback signal in the rate channel would logically explain the relative lower performance of the LQR tracking. Time-optimal (bang-bang) control achieved machine precision state tracking accuracy with the largest rate tracking error of the controllers investigated. Such performance is validated by the instinct that timeoptimal control is mathematically designed to achieve the desired state in shortest time but is not structured to simultaneously achieve rate tracking in minimal time. Cost was the lowest of controllers investigated indicating the benefits of not requiring simultaneous rate tracking. Computational run-time was the second largest. 


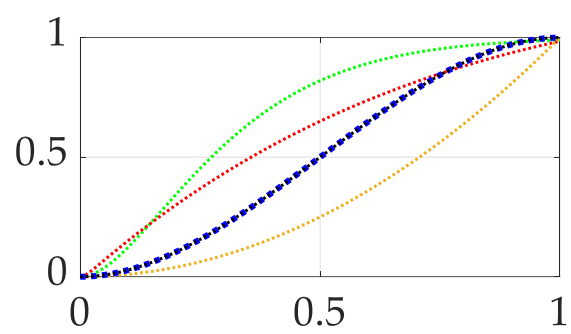

(a)

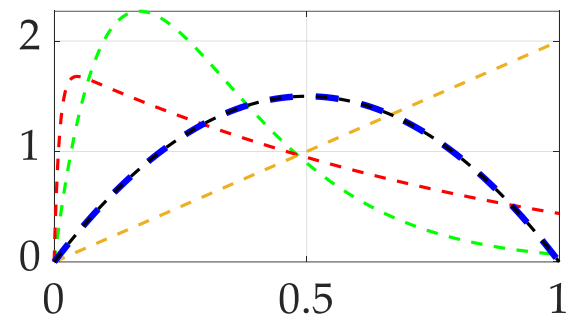

(b)

Table 7. Double-integrator plant (no transport theorem) with control design based off double-integrator

\begin{tabular}{ccccc}
\hline Method & State Error & Rate Error & Cost & Runtime \\
\hline Classical P+V & 0.010115 & 0.066169 & 28.1671 & 3.1012 \\
LQR Optimal PD & 0.015015 & 0.43861 & 76.3418 & 2.4597 \\
Time-optimal control $^{\text {Open loop optimal }}{ }^{1}$ & eps & 2 & 2 & 2.9038 \\
Ops $^{1}$ & eps & eps & 6 & 2.6086 \\
Real-time optimal (RTOC) $^{1}$ & $-9.1882 \times$ 10 $^{-6}$ & 0.019289 & 6.7656 & 2.7497 \\
Switched RTOC $^{1}$ & eps & eps & 6 & 2.7281 \\
\hline
\end{tabular}

${ }^{1}$ Real-time optimal control (with and without switching) and open loop optimal control are visually indistinguishable from one another in the graphic depiction.

(c)

Figure 2. Double-integrator plant (no transport theorem) with control design based off double-integrator. Sub-figure (a) displays the motion states (translation or rotational) normalized to propagate from zero and unity in one normalized second. Sub-figure (b) displays the motion rates (translation or rotational) intended to propagate from zero initial velocity to zero velocity at the endpoint in one normalized second. Sub-figure (c) displays the quantitative comparative data corresponding to the qualitative display in sub-figures (a) and (b).

Open-loop optimization calculates the minimum control effort that simultaneously meets state and rate endpoint conditions, and accordingly, both state and rate endpoints are achieved to machine precision, while computational burden is modest compared to low and high cases. Real-time optimization solves the open-loop optimal control problem real-time using ideal sensor feedback of state and rate but involves a matrix inverse. Rate and state errors (particularly) are quite small, but machine precision tracking is not achieved. Part of the cause of tracking errors is the inversion of rank-deficient matrix as the final time is approached. Seeking to ameliorate the issue, switched real-time optimal control is presented where the matrix condition is used to switch away from real-time optimal control to open-loop optimal control during timesteps when matrix inversion becomes poorly conditioned. Machine precision tracking is attained, open-loop optimally low costs are re-achieved, and computational burden is slightly elevated compared to the best case investigated.

Summarizing the results so far, real-time optimal control (designed only to minimize control effort) with singular switching to counter the deleterious effects of poor matrix conditioning achieves the best simultaneous state and rate error (machine precision) with costs matching the open-loop minimal and average computational burden. Unfortunately, these results are achievable only in idealized circumstances of double integrators. Expressing motion in coordinates of rotating reference frames introduced nonlinear coupling described in the next section (3.2).

\subsection{Nonlinear plants with cross-product coupled transport theorem with linear control designs}

Expressing motion in coordinates of rotating reference frames is referred to as the "transport theorem" which introduces nonlinear coupling between the six channels of motion that would otherwise have been well described by simple, linear doubleintegrators. Very often, linearized system equations or linear assumptions (e.g., the double-integrators) are used to design linear controllers. Accordingly, each instance investigated in section 3.1 was applied to nonlinear coupled system equations including the transport theorem. Increased errors and reduced robustness is generally anticipated, since the controllers are not designed to accommodate system nonlinearities specifically. Each control design technique introduced in section 2 was sequentially, and the results 
are presented in figure 3 , where table 1 is embedded as a sub-figure containing quantitative results corresponding to the qualitative results presented in the multi-plots.

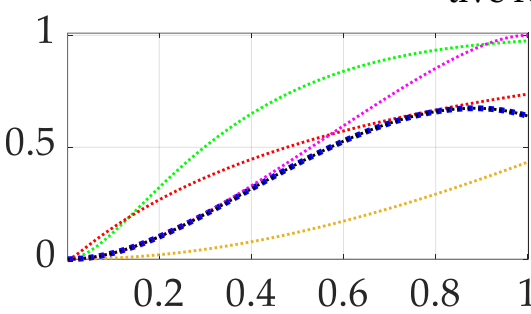

(a)

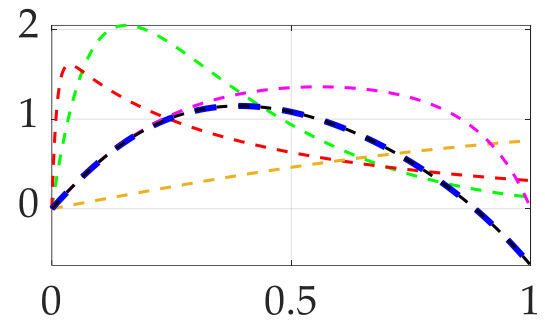

(b)

Table 8. Double-integrator plant (with transport theorem) with control design based off double-integrator (without transport theorem)

\begin{tabular}{ccccc}
\hline Method & State Error & Rate Error & Cost & Runtime \\
\hline Classical P+V & 0.024582 & 0.12803 & 26.6076 & 3.4291 \\
LQR Optimal PD & 0.26241 & 0.31372 & 75.8051 & 3.4593 \\
Time-optimal control $^{1}$ & 0.56622 & 0.76159 & 0.5 & 3.4437 \\
Open loop optimal $^{1}$ & 0.3606 & -0.63176 & 6 & 3.5315 \\
Real-time optimal (RTOC) $^{1}$ & $-1.9654 \times$ 10 $^{-5}$ & 0.041323 & 11.2002 & 3.4579 \\
Switched RTOC $^{1}$ & 0.3606 & -0.63176 & 6 & 3.5097 \\
\hline
\end{tabular}

${ }^{1}$ Real-time optimal control (with and without switching) and open loop optimal control are visually indistinguishable from one another in the graphic depiction.

(c)

Figure 3. Double-integrator plant (no transport theorem) with control design based off double-integrator. Sub-figure (a) displays the motion states (translation or rotational) normalized to propagate from zero and unity in one normalized second. Sub-figure (b) displays the motion rates (translation or rotational) intended to propagate from zero initial velocity to zero velocity at the endpoint in one normalized second. Sub-figure (c) displays the quantitative comparative data corresponding to the qualitative display in sub-figures (a) and (b).

Foremost, notice all approaches designed to control double integrators illustrate degraded performance compared to the idealized case investigated in section 3.1. All methods compared achieved similar costs. The baseline approach (classical proportional plus velocity, or $\mathrm{P}+\mathrm{V}$ ) tuned to performance specification exhibits second-best state accuracy, second-best rate accuracy, while real-time optimal control achieved the lowest state and rate errors but using nearly double the amount of control. No technique achieved machine precision tracking.

Summarizing the results so far, all control techniques are degraded from the idealized case. Real-time optimal control (designed only to minimize control effort) and classical control methods were the most robust, but all three methods utilized substantially more control effort.

\subsection{Nonlinear plants with cross-product coupled transport theorem \& nonlinear control designs}

Double integrator relationships (implemented identically as done in sections 3.1 and 3.2) are next augmented with feedback decoupling of the transport theorem using state feedback in equation (19). Each control design technique introduced in section 2 was sequentially used to control the nonlinear, double integrator system equations including transport theorem, and the results are presented in figure 3, where table 1 is embedded as a sub-figure containing quantitative results corresponding to the qualitative results presented in the multi-plots.

All methods are improved by addition of the nonlinear decoupling control designed for the transport theorem. Time optimal control performs worst regarding state and rate errors, while cost figures are generally increased for methods that effectively track state and rate. Near machine-precision is achieved by open-loop optimal control and switched, real-time optimal control where both are designed to minimize control effort alone (with no state error representation in the minimized cost function). Computational burdens of all approaches are roughly comparable. 


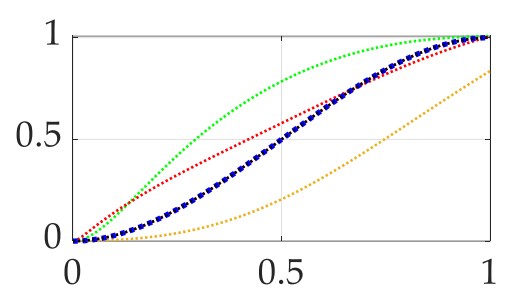

(a)

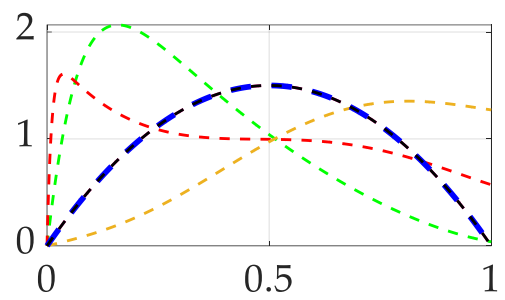

(b)

Table 9. Double-integrator plant (with transport theorem) with control design based off double-integrator with transport theorem

\begin{tabular}{ccccc}
\hline Method & State Error & Rate Error & Cost & Runtime \\
\hline Classical P+V & 0.0078728 & 0.038016 & 27.0064 & 3.5535 \\
LQR Optimal PD & -0.0063144 & 0.57121 & 75.7706 & 3.5738 \\
Time-optimal control $^{1}$ & 0.16359 & 1.2712 & 2.7286 & 3.629 \\
Open loop optimal $^{1}$ & $3.0287 \times 10^{-13}$ & $-1.0092 \times 10^{-12}$ & 7.0286 & 3.4504 \\
Real-time optimal (RTOC) $^{1}$ & $-9.1882 \times \mathbf{1 0}^{-6}$ & 0.019288 & 7.7942 & 3.6725 \\
Switched RTOC $^{1}$ & $3.0287 \times 10^{-13}$ & $-1.0092 \times 10^{-12}$ & 7.0286 & 3.5765 \\
\hline
\end{tabular}

${ }^{1}$ Real-time optimal control (with and without switching) and open loop optimal control are visually indistinguishable from one another in the graphic depiction.

Figure 4. Double-integrator plant (no transport theorem) with control design based off double-integrator. Sub-figure (a) displays the motion states (translation or rotational) normalized to propagate from zero and unity in one normalized second. Sub-figure (b) displays the motion rates (translation or rotational) intended to propagate from zero initial velocity to zero velocity at the endpoint in one normalized second. Sub-figure (c) displays the quantitative comparative data corresponding to the qualitative display in sub-figures (a) and (b).

Having initially analyzed idealized systems (i.e., the double integrators), nonlinear coupling was induced by the transport theorem with significantly degraded performance using the controllers designed for linear systems. Adding nonlinear control components designed specifically to decouple the transport theorem in feedback roughly restores nominal performances, but feedback remains ideal (without noise). Section 3.4 adds zero-mean Gaussian noise to both sensor types (state and rate)

\subsection{Nonlinear plants with cross-product coupled transport theorem \& nonlinear control designs utilizing noisy, mixed-sensors}

Double integrator equations with nonlinearities induced by transport theorem were controlled by linear control designs augmented with nonlinear feedback decoupling designed specifically for transport theorem. Feedback was provided by simulated mixed state and rate sensors with Gaussian noise added. Each control design technique introduced in section 2 was sequentially used to control the linear, double integrator system equations, and the results are presented in figure 3 , where table 1 is embedded as a subfigure containing quantitative results corresponding to the qualitative results presented in the multi-plots.

The baseline approach (classical proportional plus velocity, or $\mathrm{P}+\mathrm{V}$ ) tuned to performance specification exhibits better rate accuracy and lower costs than linear quadratic optimal regulators of the proportional, derivative (PD) type, but the $\mathrm{P}+\mathrm{V}$ controller has relatively inferior state errors compared to LQR. Time optimal control performs poorly in the face of transport theorem and noisy sensors. Real-time optimal control is severely degraded by noise, particularly with respect to rate errors and control effort.

Open-loop optimization and real-time optimal control with singular switching simultaneously achieve lowest state and rate endpoint errors, with lowest costs (that may be claimed to meet endpoint conditions), while computational burden is modest compared to low and high cases. 


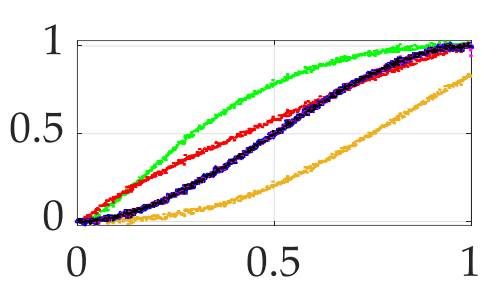

(a)

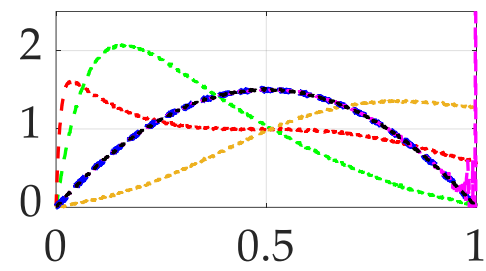

(b)

Table 10. Double-integrator plant (with transport theorem) with control design based off double-integrator with transport theorem and noisy, mixed sensors (state and rate)

\begin{tabular}{ccccc}
\hline Method & State Error & Rate Error & Cost & Runtime \\
\hline Classical P+V & -0.0066807 & 0.03828 & 27.0755 & 2.3988 \\
LQR Optimal PD & -0.0047636 & 0.56965 & 76.8806 & 2.4667 \\
Time-optimal control $^{1}$ & 0.16546 & 1.2693 & 2.7286 & 2.4837 \\
Open loop optimal $^{1}$ & 0.0018665 & -0.0018665 & 7.0286 & 2.5816 \\
Real-time optimal (RTOC) $^{1}$ & 0.06463 & -171.6553 & 41436948 & 2.469 \\
Switched RTOC $^{1}$ & 0.0018665 & -0.0018665 & 7.0286 & 2.6125 \\
\hline
\end{tabular}

${ }^{1}$ Real-time optimal control (with and without switching) and open loop optimal control are visually indistinguishable from one another in the graphic depiction.

(c)

Figure 5. Double-integrator plant (with transport theorem) with control design based off double-integrator (with transport theorem). Sub-figure (a) displays the motion states (translation or rotational) normalized to propagate from zero and unity in one normalized second. Sub-figure (b) displays the motion rates (translation or rotational) intended to propagate from zero initial velocity to zero velocity at the endpoint in one normalized second. Sub-figure (c) displays the quantitative comparative data corresponding to the qualitative display in sub-figures (a) and (b). Notice the openloop optimal (minimum) control effort is increased from 6.0 (controlling double integrators without transport theorem) to just over 7.0 (controlling double integrators with transport theorem) manifest as a $17 \%$ increase to account for transport theorem over idealized cases of double-integrators alone.

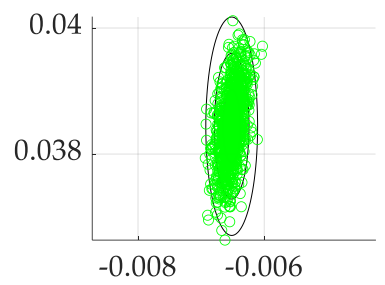

(a)

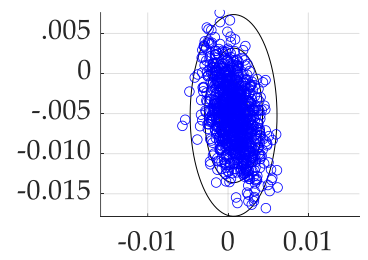

(d)

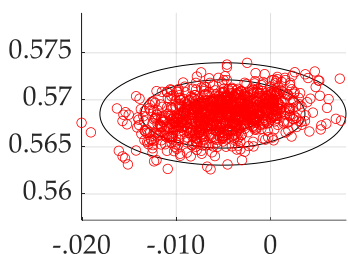

(b)

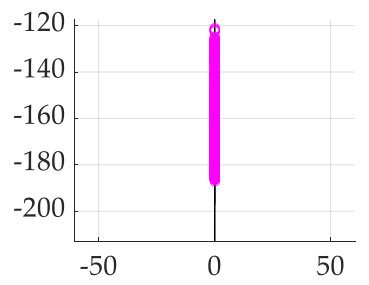

(e)

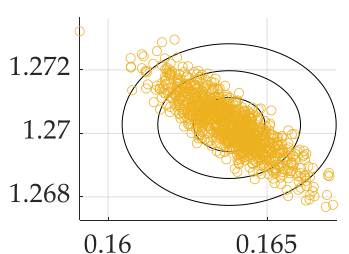

(c)

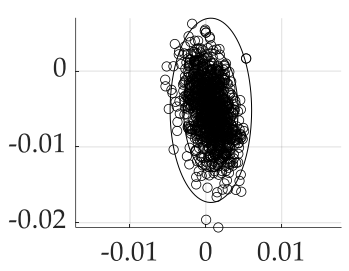

(f)

Figure 6. Scatter plots displaying the results of 1,000 simulation runs (per case) with randomly (uniformly) varied mass and mass moments $\pm 10 \%$. (a) Classical P $+V$, (b) LQR Optimal PD, (c) Time-optimal control, (d) Open loop optimal, (e) Real-time optimal (RTOC), (f) Switched RTOC.

\subsubsection{Monte Carlos analysis (6,000 simulation runs)}

Summarizing the results so far, real-time optimal control (designed only to minimize control effort) with singular switching to counter the deleterious effects of poor matrix conditioning achieves the best simultaneous state and rate error with costs matching the open-loop minimal and average computational burden in cases where nonlinear feedback decoupling of transport theorem is incorporated where feedback is provided by noisy state and rate sensors. 
Table 11. One-thousand-run (respectively) Monte Carlo analysis.

\begin{tabular}{ccccc}
\hline Method & State Error & Rate Error & Cost & Runtime \\
\hline Classical P+V & -0.0065157 & 0.038442 & 25.3181 & 1.2342 \\
LQR Optimal PD & -0.0050504 & 0.56851 & 75.5278 & 1.23 \\
Time-optimal control & 0.16381 & 1.2703 & 1.3643 & 1.3055 \\
Open loop optimal $^{1}$ & 0.00069197 & -0.0052251 & 4.0281 & 1.2335 \\
Real-time optimal (RTOC) $^{1}$ & 0.062551 & -165.1258 & 40959.5421 & 1.2818 \\
Switched RTOC $^{1}$ & $\mathbf{0 . 0 0 0 6 6 1 1 7}$ & $\mathbf{- 0 . 0 0 5 1 7 4 6}$ & $\mathbf{4 . 0 2 8 1}$ & $\mathbf{1 . 1 0 6 8}$ \\
\hline
\end{tabular}

${ }^{1}$ Real-time optimal control (with and without switching) and open loop optimal control are visually indistinguishable from one another in the graphic depiction.

\section{Discussion}

The results are multi-variate, but some general comments are evident regarding the proposed real-time optimal control with singular switching and transport theorem decoupling and its performance compared to a classical benchmark and four other instantiations of optimal control. In the most realistic situations revealed by Monte Carlo analysis with random variations of inertia and state and rate sensor noise, time-optimal bang-bang control achieved respectable rate accuracy with the lowest cost but highest run-time and modest rate tracking errors. Meanwhile optimal (control minimizing constrained to meet endpoint conditions) open-loop control and its companion real-time optimal control with singular switching achieved the lowest state errors (three orders of magnitude better than time-optimal control) and control effort, while real-time optimal control with singular switching and transport theorem decoupling achieved the lowest rate tracking error. Real-time optimal control without singular switching displayed vulnerability in rate errors and high costs.

Other general conclusions apply to all techniques: designing controls based on simplified plants, and then applying them to realistic plants is particularly weak compared to the relatively more modern approaches. Arguably, milli-degree accuracy with "low" costs is admirable performance, but the modern methods of control design including optimality and nonlinear coupling effects (with feedback) achieved in general, three orders of magnitude superior performance, with the admission that real-time optimal control performed particularly poorly.

Furthermore, well known lessons from classical control are re-validated in this study. Linear-quadratic regulators are very robust and useful, but suffer from cascaded topologies, particularly in the differentiation of the state feedback to achieve rate feedback, thus the utilization of velocity control was established as the classical baseline (with a requisite demand to purchase and utilize rate sensors).

The proposed instantiation of real-time optimal control with singular switching and transport theorem decoupling was the overall top performing option with the lowest state errors, lowest rate errors, lowest computational burden, and second-lowest control effort (i.e., fuel usage).

Lastly, notice all the control techniques performed very well (naturally, since most of the techniques were formulated to satisfy optimization problems). The indication of superior performance should not be judged as mandating the proposed technique, especially in instance where operators would be more comfortable with classical techniques and the order of milli-degree accuracy is sufficient.

\subsection{Performance improvement percentages}

The claim was just immediately earlier validating that real-time optimal control with singular switching and transport theorem decoupling was the overall top performing option, and this section describes the results validating the claim in generally understandable terms (percent performance improvement comparison). Open loop (control minimizing optimal control constrained to meet end state and rate) performed very well, while real-time optimal control with singular switching matched the performance and was slightly better in terms of computational burden. 
Table 12. Percentage change in performance in one-thousand-run (respectively) Monte Carlo analysis: Double-integrator plant (with transport theorem) with control design based off doubleintegrator with transport theorem and noisy, mixed sensors (state and rate).

\begin{tabular}{ccccc}
\hline Method & State Error & Rate Error & Cost & Runtime \\
\hline Classical P+V & -- & -- & -- & -- \\
LQR Optimal PD & $-22 \%$ & $1379 \%$ & $198 \%$ & $0 \%$ \\
Time-optimal control & $2614 \%$ & $3204 \%$ & $-95 \%$ & $6 \%$ \\
Open loop optimal ${ }^{1}$ & $-111 \%$ & $-114 \%$ & $-84 \%$ & $0 \%$ \\
${\text { Real-time optimal (RTOC) }{ }^{1}}_{\text {Switched RTOC }}{ }^{1}$ & $1060 \%$ & $429645 \%$ & $161680 \%$ & $4 \%$ \\
\hline
\end{tabular}

${ }^{1}$ Real-time optimal control (with and without switching) and open loop optimal control are visually indistinguishable from one another in the graphic depiction.

\subsection{Future research}

The derivation of optimal trajectories (state, rate, acceleration, jerk, etc.) should prove useful in the implementation of deterministic artificial intelligence [28] which requires some scheme of autonomous trajectory generation. The current state of the art utilizes sinusoidal trajectory generation schemes, and the optimal trajectories illustrated here should have improved efficacy when used to augment deterministic artificial intelligence.

Funding: This research received no external funding. The APC was funded by the corresponding author.

Data Availability Statement: All data supporting reported results can be obtained by contacting the corresponding author.

Conflicts of Interest: The authors declare no conflict of interest. 


\section{Appendix A}

The appendix contains details of the simulations, first with SIMULINK topologies, and secondly with MATLAB line-code to aid the readership replicate the experiments presented in this manuscript.

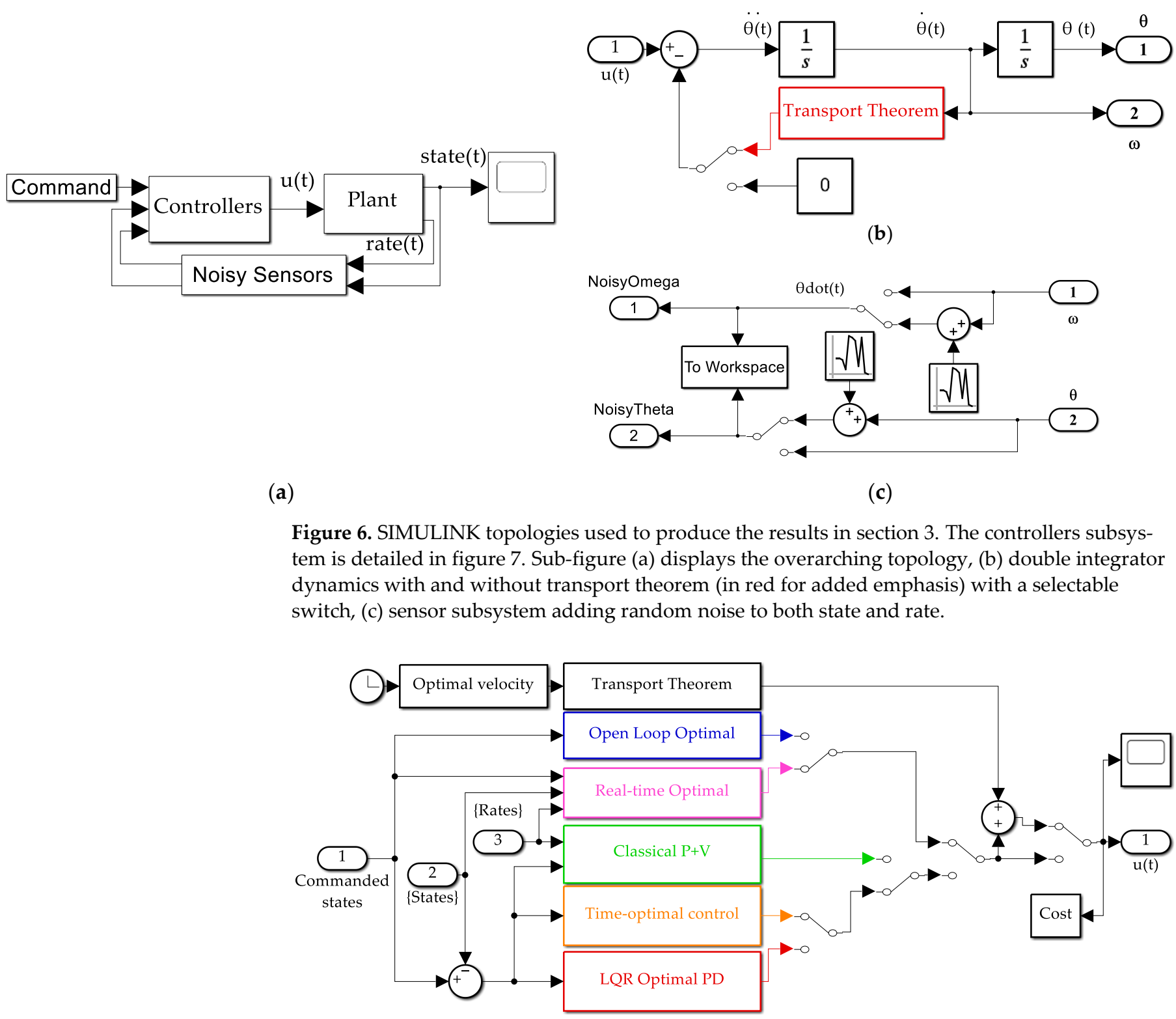

Figure 7. SIMULINK model for controllers where manual switches are used to evaluate each technique in direct comparison where all other facets are held constant except the control methodology used. The coloring of the blocks in the figure are consistent with the coloring of the data lines plotted in subsequent figures throughout section 3 of the manuscript. Red coloring indicates linear-quadratic regulator with proportional derivative control, orange indicates time-optimal control. Green color indicates proportional plus velocity $(\mathrm{P}+\mathrm{V})$ control. Pink indicates real-time optimal control, blue indicates open loop optimal control, and black indicates transport theorem control components. 


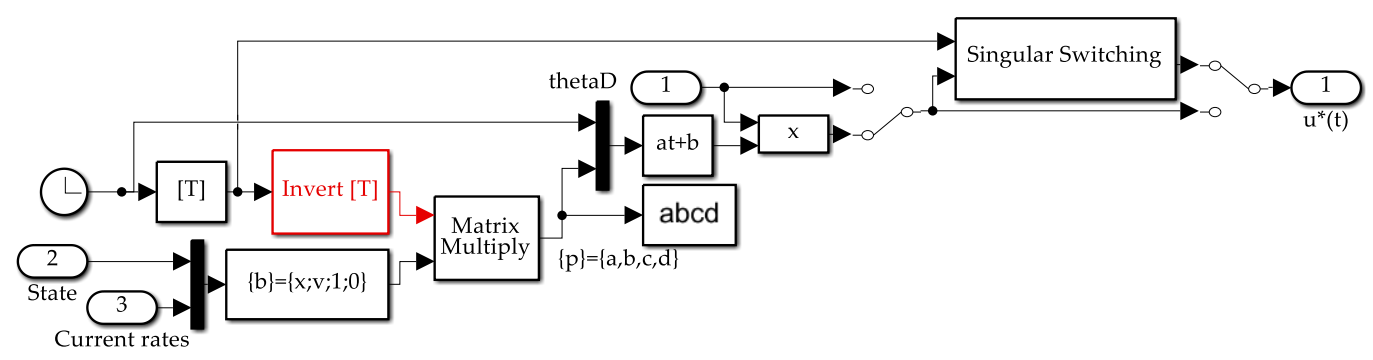

Figure 8. SIMULINK model implementing real-time optimal control with and without singular switching (manually selectable using the switch on the right-hand side of the simulation graphic). The red simulation block labeled "Invert [T]" is displayed in figure 9.

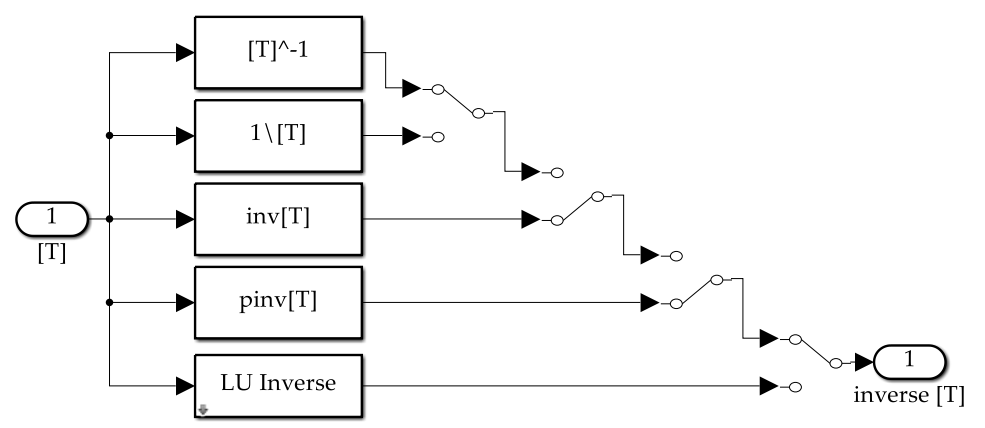

Figure 9. Simulation blocks implementing options for matrix inversion in real-time optimal control.

clear all; close all; clc; warning off;

ENDSTATE=[]; COSTEND=[]; TICTOC=[]; COST=[];

for $\mathrm{i}=1: 1000$; tic; i

sim('IterateOnOptimalControl'); toc;

EndState=States(end,:); ENDSTATE=[ENDSTATE; EndState];

COSTEND=[COSTEND; Cost(end)]; TICTOC=[TICTOC; toc];

COST $=[\operatorname{COST}$; squeeze(Cost)];

end

$\mathrm{C}=[1$-mean(ENDSTATE(:,1)) mean(ENDSTATE(:,2)) $;$ \% ellipse center $\mathrm{a}=\operatorname{std}(1-\operatorname{ENDSTATE}(:, 1)) ; \mathrm{b}=\operatorname{std}(\operatorname{ENDSTATE}(:, 2)) ; \%$ major and minor axis hold on; $\operatorname{plot}\left(\mathrm{C}(1)+\mathrm{a}^{*} \cos \left(\right.\right.$ linspace $\left.\left(0,2^{*} \mathrm{pi}\right)\right), \mathrm{C}(2)+\mathrm{b}^{*} \sin \left(\right.$ linspace $\left.\left.\left(0,2^{*} \mathrm{pi}\right)\right), \mathrm{\prime}^{\prime}\right)$; $\operatorname{plot}\left(\mathrm{C}(1)+2^{*} \mathrm{a}^{*} \cos \left(\right.\right.$ linspace $\left.\left(0,2^{*} \mathrm{pi}\right)\right), \mathrm{C}(2)+2^{*} \mathrm{~b}^{*} \sin \left(\right.$ linspace $\left.\left.\left(0,2^{*} \mathrm{pi}\right)\right), \mathrm{\prime}^{\prime} \mathrm{k}\right)$; $\operatorname{plot}\left(\mathrm{C}(1)+3^{*} \mathrm{a}^{*} \cos \left(\right.\right.$ linspace $\left.\left(0,2^{*} \mathrm{pi}\right)\right), \mathrm{C}(2)+3^{*} \mathrm{~b}^{*} \sin \left(\right.$ linspace $\left.\left.\left(0,2^{*} \mathrm{pi}\right)\right), \mathrm{k}^{\prime}\right)$; scatter(1-ENDSTATE(:,1), ENDSTATE(:,2)); grid; axis equal; set(gca,'fontsize',18, 'fontname','palatino linotype');

LEGENDTEXT1=['\mu TicToc $=$ ' num2str(mean $($ TICTOC $))]$

LEGENDTEXT2=['; \mu StateError = ' num2str(1-mean(ENDSTATE(:,1)))]

LEGENDTEXT3=['; \mu RateError = ' num2str(mean(ENDSTATE(:,2)))]

LEGENDTEXT4=['; \mu Cost = ' num2str(mean(COST))] LEGENDTEXT=[LEGENDTEXT1 LEGENDTEXT2 LEGENDTEXT3 LEGENDTEXT4] legend(LEGENDTEXT)

hold off; clc; 


\section{References}

1. O'Brien, J. Solar Induced Fluorescence: Learn a New Approach to Remote Sensing of Vegetation. Explore Earth, website of The Applied Sciences Program is part of the Earth Science Division of the NASA Science Mission Directorate. Available online, accessed December 23, 2021, at https://appliedsciences.nasa.gov/our-impact/news/solar-induced-fluorescence-learn-newapproach-remote-sensing-vegetation.

2. NASA image use policy. Available online, accessed December 23, 2021, at https://gpm.nasa.gov/image-use-policy

3. Chaia,R.; Savvarisa, A.; Tsourdosa, A.; Tsourdos, A.; Chai, S.; Xia, Y. A review of optimization techniques in spacecraft flight trajectory design. Progress in Aerospace Sciences 2019, 109(2). Doi: 10.1016/j.paerosci.2019.05.003.

4. Rao, A.; Tang, S.; Hallman, W. Numerical optimization study of multiple-pass aero assisted orbital transfer, Optimal Control Applications and Methods 2002, 23(4), 215-238. doi:10.1002/oca.711.

5. Rao, A. A survey of numerical methods for optimal control, Advances in the Astronautical Sciences 2009, 135(1), 497-528.

6. Ross, I. M.; Karpenko, M. A review of pseudospectral optimal control: From theory to flight, Annual Reviews in Control 2012, 36(2), 182-197. doi: https://doi.org/10.1016/j.arcontrol.2012.09.002.

7. Gao, H.; Yang, X.; Shi, P. Multi-objective robust h-infinity control of spacecraft rendezvous, IEEE Transactions on Control Systems Technology 2009, 17(4) 794-802. doi:10.1109/TCST.2008.2012166

8. Bonnans, J.; Festa, A. Error estimates for the Euler discretization of an optimal control problem with first-order state constraints, SIAM Journal on Numerical Analysis 2017, 55(2) 445-471. doi:10.1137/140999621.

9. Pontani, M.; Conway, B. Optimal finite-thrust rendezvous trajectories found via particle swarm algorithm, Journal of Spacecraft and Rockets 2013, 50(6), 1222-1234. doi:10.2514/1.A32402.

10. Gan, C.; Zi-ming, W.; Min, X.; Si-lu, C. Genetic Algorithm Optimization of RLV Reentry Trajectory, In Proceedings of the AIAA/CIRA $13^{\text {th }}$ International Space Planes and Hypersonic Systems and Technologies Conference, Capua, Italy, 16-20 May 2005. doi:10.2514/6.2005-3269.

11. Kenan, Z; Wanchun, C. Reentry Vehicle Constrained Trajectory Optimization, In Proceedings of the 17th AIAA International Space Planes and Hypersonic Systems and Technologies Conference, San Francisco, California, 11-14 April 2011. doi:10.2514/6.2011-2231.

12. Rajesh, A. Reentry Trajectory Optimization: Evolutionary Approach, In Proceedings of the 9th AIAA/ISSMO Symposium on Multidisciplinary Analysis and Optimization, Atlanta, Georgia, 4-6 September 2002. doi:10.2514/6.2002-5466.

13. Robert, W.; Mark, A. Jeffrey, B.; Robert, W.; Mark, A.; Jeffrey, B. Minimum heating reentry trajectories for advanced hypersonic launch vehicles, In Proceedings of the Guidance, Navigation, and Control Conference, New Orleans, LA, U.S.A. 11-13 August 1997. doi: 10.2514/6.1997-3535.

14. Mikhail, I.; Pavel, V.; Alexandr, K. Numerical Investigation of the EXPERT Reentry Vehicle Aerothermodynamics Along the Descent Trajectory, In Proceedings of the 39th AIAA Thermophysics Conference, Miami, Florida, $25-28$ June 2007. doi:10.2514/6.2007-4145.

15. Ross, M. A primer on Pontryagin's principle in optimal control, CA: Collegiate Publishers, 2015.

16. Tian, B.; Fan, W.; Su, R.; Zong, Q. Real-time trajectory and attitude coordination control for reusable launch vehicle in reentry phase, IEEE Transactions on Industrial Electronics 2015, 62(3), 1639-1650. doi:10.1109/TIE.2014.2341553.

17. Sagliano, M.; Mooij, E.; Theil, S. Onboard trajectory generation for entry vehicles via adaptive multivariate pseudospectral interpolation, Journal of Guidance, Control, and Dynamics 2017, 40(2), 466-476. doi:10.2514/1.G001817.

18. R. Chai, A. Savvaris, A. Tsourdos, S. Chai, Y. Xia, Optimal tracking guidance for aeroassisted spacecraft reconnaissance mission based on receding horizon control, IEEE Transactions on Aerospace and Electronic Systems 2018, 54(4), $1575-1588$.

19. Chai, R,; Savvaris, A.; Tsourdos, A.; Chai, S.; Xia, Y. Optimal fuel consumption finite-thrust orbital hopping of aeroassisted spacecraft, Aerospace Science and Technology 2018, 75, 172-182. doi:https://doi.org/10.1016/j.ast.2017.12.026.

20. Willems, J. Least squares stationary optimal control and the algebraic Riccati equation. IEEE Trans. Aut. Control, 1971, 16(6), pp. 621-634.

21. Kwakernaak, H.; Sivan, R. Linear Optimal Control Systems. First Edition. Wiley-Interscience, Hoboken, USA, 1972.

22. Kelly, R. Proportional Control plus Velocity Feedback and PD Control. In Control of Robot Manipulators in Joint Space. Kelly, R.; Davila, V.; Loría, A.; Advanced Textbooks in Control and Signal Processing. Springer, London, 2005; pp 141-156. https://doi.org/10.1007/1-85233-999-3_8 Publisher: Publisher Location, Country, 2007; Volume 3, pp. $154-196$.

23. Flugge-Lotz, I. Discontinuous Automatic Control. Princeton University Press: Princeton, USA, 1953.

24. Sands, T. Kim, J., Agrawal, B. Improved Hamiltonian Adaptive Control of spacecraft, In Proceedings of the IEEE Aerospace Conference, Big Sky, MT, USA 2009, March 7-14, 2009. doi: 10.1109/AERO.2009.4839565.

25. Sands, T. Comparison and Interpretation Methods for Predictive Control of Mechanics. Algorithms 2019, 12(11), 232

26. Sands, T. Physics-Based Control Methods, In Advances in Spacecraft Systems and Orbit Determination, Ghadawala, R. (editor); IntechOpen: London, U.K., 2011. DOI: 10.5772/36128. Available from: https://www.intechopen.com/chapters/33681.

27. https://www.engr.wisc.edu/news/robert-lorenz-pioneer-controls-engineering-passes-away/

28. Smeresky, B.; Rizzo, A.; Sands, T. Optimal Learning and Self-Awareness Versus PDI. Algorithms 2020, 13(1), 23. https://doi.org/10.3390/a13010023

29. Baker, K.; Cooper, M.; Heidlauf, P.; Sands, T. Autonomous trajectory generation for deterministic artificial intelligence. Electr. Electron. Eng. 2018, 8(3), 59. 
30. Heidlauf, P.; Cooper, M. "Nonlinear Lyapunov Control Improved by an Extended Least Squares Adaptive Feed Forward Controller and Enhanced Luenberger Observer", In Proceedings of the International Conference and Exhibition on Mechanical \& Aerospace Engineering, Las Vegas, NV, USA, 2-4 October 2017.

31. Pontryagin, L.; Boltayanskii, V.; Gamkrelidze, R.; Mishchenko, E. The mathematical theory of optimal processes, Wiley Interscience, New York, 1962. (Translated from Russian)

32. Boltyanskii, V. Mathematical methods of optimal control, Holt, Rinehart \& Winston, 1971. (Translated from Russian)

33. Sands, T. Virtual Sensoring of Motion Using Pontryagin's Treatment of Hamiltonian Systems. Sensors 2021, $21(13), 4603$. https://doi.org/10.3390/s21134603

34. Kinematics Handout - MIT OpenCourseWare. Available online, accessed 30 December 2021: https://ocw.mit.edu/courses/mechanical-engineering/2-003sc-engineering-dynamics-fall-2011/newton2019s-laws-vectors-andreference-frames/MIT2_003SCF11Kinematic.pdf

35. CalTech notes, Chapter 2 Optimization-based control. Available online, accessed 30 December 2021: https://www.cds.caltech.edu/ murray/books/AM08/pdf/obc-optimal_04Jan10.pdf 